\title{
Meta
}

Journal des traducteurs

Translators' Journal

\section{LETEINTURIER, C. (1990) : Dictionnaire multimédia, Presse, Radio, Télévision, Publicité..., Paris, Eyrolles, 121 p.}

\section{Louise Brunette}

Volume 36, numéro 2-3, juin 1991

URI : https://id.erudit.org/iderudit/002169ar

DOI : https://doi.org/10.7202/002169ar

Aller au sommaire du numéro

Éditeur(s)

Les Presses de l'Université de Montréal

ISSN

0026-0452 (imprimé)

1492-1421 (numérique)

Découvrir la revue

Citer ce compte rendu

Brunette, L. (1991). Compte rendu de [LETEINTURIER, C. (1990) : Dictionnaire multimédia, Presse, Radio, Télévision, Publicité..., Paris, Eyrolles, 121 p.] Meta, 36(2-3), 524-526. https://doi.org/10.7202/002169ar

Ce document est protégé par la loi sur le droit d'auteur. L'utilisation des services d'Érudit (y compris la reproduction) est assujettie à sa politique d'utilisation que vous pouvez consulter en ligne.

https://apropos.erudit.org/fr/usagers/politique-dutilisation/
Cet article est diffusé et préservé par Érudit.

Érudit est un consortium interuniversitaire sans but lucratif composé de l’Université de Montréal, l'Université Laval et l'Université du Québec à Montréal. Il a pour mission la promotion et la valorisation de la recherche. https://www.erudit.org/fr/ 
- LETEINTURIER, C. (1990): Dictionnaire multimédia, Presse, Radio, Télévision, Publicité..., Paris, Eyrolles, 121 p.

Le vocabulaire des médias n'a jamais fait, dans son ensemble, l'objet d'une étude lexicographique ou terminologique. On comprend pourquoi: le champ est trop vaste, l'arbre de domaines trop ramifié. 
Pourtant, l'étendue du champ à étudier n'a pas rebuté l'auteure de ce travail «pluridisciplinaire» qui, si l'on se fie à la préface, est «un ouvrage de référence... un dictionnaire et une encyclopédie». A priori, il n'y a pas de quoi se réjouir de voir publié un travail de cette ampleur. Mais la désillusion vient vite...

Il y a un danger à faire de la terminologie sans terminologue; l'auteure ne l'a pas évité. C'est ce qui explique l'absence complète de méthode dans le traitement des 1700 termes définis.

D'abord, le domaine est mal circonscrit. La meilleure preuve en est donnée dans le titre (notez les points de suspension). D'ailleurs une consultation rapide de l'ouvrage permet de dégager un très grand nombre de sous-domaines: droit, informatique, cinéma, commerce, affichage, télécommunications, électronique, imprimerie, sondages, appellations d'emploi, appellations officielles. Soulignons, au passage, que le domaine d'emploi n'est pas donné pour chaque entrée.

Parlons du nombre des termes: 1700 . C'est bien peu si l'on considère que la nomenclature du Vocabulaire bilingue de la mesure en radiotélévision publié en 1985 par le Service de linguistique et de traduction de Radio-Canada comportait, pour le seul domaine des sondages, 1900 entrées. Ajoutons à cela qu'en 1988, Robert Dubuc publiait le Vocabulaire bilingue du droit d'auteur appliqué à l'audiovisuel traitant de plus de 300 termes. Si un ouvrage doit être exhaustif pour mériter l'appellation de dictionnaire, il faudrait songer immédiatement à modifier le titre de celui de Mme Leteinturier.

Les marques, ou leur utilisation correcte, sont à peu près inconnues de l'auteure. Ainsi, selon le caprice du moment, on trouvera une note grammaticale nous disant que câbliste (n. m.) est, on le présume, un nom masculin, tout comme bureautique (n. m.)!!! On apprend également dans cet ouvrage que serré est un adverbe (adv.)! Mais, sur le genre problématique du substantif vidéo, pas le moindre renseignement.

On cherchera sans succès dans tout l'ouvrage une seule indication de niveau de langue. Le non-initié devra, par exemple, faire des recherches pour vérifier si le terme beurre est une désignation familière ou technique en imprimerie. La même observation s'applique à SIT-COM en radiotélévision et à serpent de mer en journalisme.

Évidemment, l'auteure ne connaît pas non plus les marques géographiques. Ces marques auraient été utiles dans le cas des délits de presse définis par le code pénal français et dont les appellations ne peuvent être transposées en dehors de l'Hexagone. $\grave{A}$ ce propos, on peut quand même s'interroger sur la pertinence de définitions juridiques dans un dictionnaire... Mais ces dernières ont le mérite d'être rédigées selon les règles de l'art. On ne peut en dire autant de nombreuses autres qui ressemblent davantage à des monographies. Ainsi, à lancement d'une publication on trouve la description sur six lignes des étapes de l'opération en cause. L'entrée jeunes et médias introduit un article sur le rapport particulier des médias avec la jeunesse. D'autres définitions laissent perplexes. Ainsi, visa de contrôle cinématographique nous donne: marque l'accord du Ministre de la culture pour l'exploitation publique... et, mort kilométrique: l'intérêt du lecteur décroît avec la distance... Autre fantaisie définitoire: laissé sur le marbre, curieuse entrée, sans marque d'usage, se définit: désigne les articles composés mais finalement non imprimés. Je ne m'attarderai pas longtemps sur les définitions essentiellement douteuses comme celle de critère socio-démographique: ensemble de huit éléments... Suit une énumération de sept éléments.

Le vocabulaire des médias étant particulièrement perméable aux anglicismes, un dictionnaire multimédia doit en faire état. Mme Leteinturier l'a compris, mais elle ne définit nulle part ce qu'elle entend par anglicisme ni ne prend position face à l'usage des termes ainsi qualifiés. Cependant, on constate à la lecture que cette étiquette est donnée aux mots à graphie anglaise. Exemples : dolby, dope-sheet, prospect, copyright, playback, 
corporate. Dans certains cas, comme pour dolby, le terme est défini sans qu'on en donne un équivalent français de remplacement. Pour dope-sheet, on nous renvoie à note de tournage, cette entrée étant elle-même suivie de la mention : pour dope-sheet. Que faut-il comprendre? «Pour remplacer dope-sheet» ou «Utilisé à mauvais escient»? Mystère! Regardons aussi corporate. Ici on nous renvoie à publicité institutionnelle. Qui cherchera la définition de publicité institutionnelle à corporate? À noter à ce sujet: corporate adversiting ne figure nulle part comme entrée. Dans le même ordre d'idée, qui regardera à mensongère pour avoir la définition de publicité mensongère ou à extérieur (sic), pour avoir celle d'affichage.

Le deuxième grand défaut de l'ouvrage est le manque de tenue. Il serait trop long d'énumérer, par exemple, toutes les formes d'abréviation utilisées pour les renvois (du genre $\langle\mathrm{V} »$ ou $\langle\mathrm{V} »$ ou $\langle\mathrm{V} »$, pour voir) ou de faire la liste des fautes d'orthographe ou des incohérences dans la graphie d'un même mot.

N'y a-t-il donc rien de bon dans ce dictionnaire multimédia? Répondre «rien» serait exagérer. «Pas grand-chose» serait plus près de la vérité. Côté positif, il faut parler de la présence dans l'ouvrage de certains néologismes attestés, comme lectorat (readership) et localier (local editor). De plus, ces néologismes sont définis avec toute la rigueur qui caractérise la terminologie. Comment s'en étonner? Ils sortent tout droit du Dictionnaire des néologismes officiels du Commissariat général de la langue française publié en 1988. À mentionner aussi quelques ensembles de termes intéressants qui peuvent servir à l'étude de certaines typologies comme celle de la radio (radio périphérique, radio internationale, radio libre), celle de la publicité (publicité institutionnelle, publicité rédactionnelle, publicité politique) ou encore celle des articles de journaux (articles, papier, reportage, chronique, rubrique). Dernière note négative: on peut se demander pourquoi avoir défini dans un ouvrage déjà beaucoup trop vaste par son orientation des termes aussi connus que page, téléviseur, lecteur qui n'ont dans les domaines étudiés aucune signification technique particulière. On aurait eu intérêt à consacrer davantage de temps à donner des équivalents justes pour certains anglicismes. Ainsi, on n'aurait pas rendu off the record par hors enregistrement mais par confidentiel ou par officieux.

En quatrième de couverture, on peut lire: «Chignon, bifteack, mort kilométrique, médialogie, mercatique, vidéogramme, "zapping", B.L.I.C., C.E.S.P., Comité Technique Régional... termes techniques, néologismes, argots professionnels, sigles et organisations sont réunis pour la première fois en un corpus unique pour constituer ce dictionnaire multimédia». Il y aurait fallu dire: «réunis sans méthode». La maison Eyrolles nous avait pourtant habitués à de la qualité.

Ce dictionnaire n'en n'est pas un. Il s'agit, en fait, d'un aide-mémoire qui permet peut-être aux spécialistes des médias français de se retrouver dans leur univers touffu. Sur le plan terminologique, le Dictionnaire multimédia n'est pas sans valeur. Il constitue une excellente base de recherche, un excellent dossier de dépouillement. Là où Mme Leteinturier finit, un terminologue devrait commencer. Mais, en s'imposant ce travail, il faudra songer à reconstituer le corpus. D'ailleurs, la bibliographie ressemble au reste de l'ouvrage: elle est bien mince. Tout est à faire. 